\title{
Curative effect analysis of Shenqi two thistles Er Zhi decoction in the treatment of IgA nephropathy of qi-yin deficiency
}

\author{
Xiaoyong Chen, Zichuang Wang \\ Henan University of Traditional Chinese Medicine, Zhengzhou, 450002, China \\ hunter2011@foxmail.com
}

Keywords: IgA nephropathy, Shenqi two thistles Er Zhi decoction, Treatment

\begin{abstract}
Purpose Observed and analyzed the curative effect of Shenqi two thistles Er Zhi decoction in the treatment of IgA nephropathy of qi-yin deficiency. Method Used 80 cases of IgA nephrosis patients treated in our hospital from January 2012 to January 2014 as the research object, divided into two groups, the experimental group and the conventional group according to the random number method.40 cases of conventional group were treated with clinical treatment, including anti infection, antihypertensive therapy; 40 cases of experimental group were treated with clinic treatment of Shenqi two thistles Er Zhi decoction on the basis of the treatment of conventional group. To analyze the total effective rate, urinary red blood cell count, urine protein quantitative and so on, and compare difference of two groups. Results After clinical treatment, the total effective rate of the experimental group was $90.0 \%$, and the conventional group was $70.0 \%$, which was significantly lower than the experimental group, and there was significant difference between two groups $(\mathrm{P}<0.05)$, and the improvement of urine red blood cell count, urine protein and urine protein in the experimental group was significantly better than that in the conventional group,there was significant difference compared( $(\mathrm{P}<0.05)$. Conclusion After clinical treatment of self designed Shenqi two thistles Er Zhi decoction in the treatment of IgA nephropathy patient, the total efficiency of the patients of this group was improved obviously, and the laboratory indicators of urine red blood cell count, urine protein quantitative were improved,so it should be used as a clinical priority to the adoption of treatment, worth further promotion and application.
\end{abstract}

\section{Introduction}

IgA nephropathy is one of the common primary glomerulonephritis in clinical practice, which is called Berger ${ }^{[1]}$. The clinical manifestations of this disease are varied, and it is often associated with simple microscopic hematuria, proteinuria, hypertension, edema and renal function decline and so on $^{[2]}$, which is an important factor causing the end stage renal failure. At present, there is no specific treatment for western medicine, many treatment programs fails to achieve the desired therapeutic effect. On the basis of conventional treatment, the study adds Shenqi two thistles Er Zhi decoction, and explore its clinical application effect, the purpose is to provide a reference for the treatment of diseases, and now the information is as follows.

\section{Materials and Methods}

Base data. Used 80 cases of IgA nephrosis patients treated in our hospital from January 2012 to January 2014 as the research object, and all the patients were diagnosed with IgA nephrosis by clinical examination before the treatment, medical personnel explained the disease treatment principle, treatment methods, attention matters and so on in details to the patients and their families, got the informed consent signed by the patients and their families.At the same time, medical personnel should ask the subjective feeling of the patients in details, understand the basic situation of the patient's disease, explain the problems the patient did not know, eliminate the patient's doubts, make they actively cooperate with the disease treatment. 
Exclusion criteria:Excluding the hepatitis B-related nephritis, non glomerular hematuria (tuberculosis, calculus, tumors, urinary tract infection, etc.), lupus nephritis, anaphylactic purpura nephritis ${ }^{[3]}$, etc..

Diagnostic criteria: serum creatinine levels were below $133 \mu \mathrm{mol} / \mathrm{L}$, urine protein level was $0.5-3 \mathrm{~g} / 24 \mathrm{~h}^{[4-5]}$, and all of them were microscopic hematuria ,proteinuria, and all pathological examination were confirmed of IgA nephropathy, and pathological grade was between I -III.

According to the random number method, the experimental group and the conventional group, and 40 cases in each group. The male female ratio of patients in the experimental group was 22: 18, the oldest was 68 years old; the youngest was 18 years old; the average age was $(31.2+5.8)$ years old. The shortest sick time was 2 months; the longest was 6 years; the average sick time was $(2.3+0.2)$ years. The male female ratio of the conventional group was 21: 19; the oldest was 67 years old, the youngest was 17 years old; the average age was (31.5 5.2) years old. The shortest sick time was 3 months, the longest was 5 years, the average sick time was $(2.2+0.3)$ years. There were no significant difference in the gender, age, course of disease and other basic data of the experimental group $(\mathrm{P}>0.05)$, which had good comparability.

Clinical treatment. The patients of experimental group and conventional group both carried on clinical treatment, including anti infection, anticoagulation and anti hypertension; if a large amounts of urine protein was diagnosed, $0.4-0.6 \mathrm{mg} / \mathrm{kg}^{[6]}$ oral prednisone should be given.

Patients in the experimental group carried on the clinical treatment with adding self-made Shenqi two thistles Er Zhi decoction on that basis. Prescription composition including:astragali radix 40g, radix pseudostellariae $30 \mathrm{~g}$, early eclipta prostrata $30 \mathrm{~g}$, madder $20 \mathrm{~g}$, circium japonicum $15 \mathrm{~g}$, herba cepbalanoplosis $15 \mathrm{~g}$, glossy privet fruit $15 \mathrm{~g}$, gorgon fruit $15 \mathrm{~g}$, fructus rosae laevigatae $15 \mathrm{~g}$, the root of fangji $10 \mathrm{~g}$, radix achyranthis bidentatae $10 \mathrm{~g}$. (The dosage was behind the piperzine.)

If the patients had hypertension complicated headache, uncaria, gastrodia elata could be added into; if patients had a significant blood stasis, centella, ligusticum wallichii, the root of red-rooted salvia could be used; if the patients had constipation, cistanche, and prepared rhubarb can be added; if edema of lower extremity of the patients, the herba plantain and the shell of the calabash were good for it; if patients with the mouth swallows dry does, redix ophiopogon and dendrobe did well. Total water for clothing daily 3 agent for 3 months, then, compared the clinical treatment effect of the patients of two groups.

Evaluation index. Compared the index of laboratory examination of the experimental group and the conventional group before and after the treatment, such as: blood urea nitrogen, serum creatinine, urine red blood cell count, urine protein, etc.. At the same time, carried on the reexamination of routine urine routine examination of patients every week, and the reexamination of the urine protein every 2 weeks, and the reexamination of renal function of the patients every month.

The criteria of therapeutic effects. Compared clinical treatment effect of patients of two groups, all with reference to the judgement standard of IgA nephropathy in The Guiding Principle of Clinical Research of New Traditional Chinese Medicine ${ }^{[7]}$, and the clinical treatment effect can be divided into complete remission, significant response, improvement, invalid the four criteria. (1) complete remission: clinical symptoms and signs disappeared; the extinction time of red blood cell was 3-4 months; urine protein check was negative; (2) significant response:the clinical symptoms and signs were significantly relieved, and red blood cells and urine protein decreased more than $50 \%$; (3) improvement:the renal function returned normal. and red blood cells and urine protein decreased more than 25\%;(4)invalid:no improvement or even worse.

Data analysis. The clinical data of patients with IgA nephropathy in this study were recorded to SPSS18.0 software, and the clinical treatment effect was represented with percentage and ratio, compared U-RBC, $24 \mathrm{~h}$ urine protein quantitation, $\mathrm{SCr}$ with $\mathrm{X} 2$ test, and the level of BUN was expressed with standard deviation \pm of sample means $(\bar{\chi} \pm S)$; T was comparison test; $\mathrm{P}<0.05$ was thought that the difference had statistical significance. 


\section{Results}

Compared the clinical effect of the patient of the experimental group and the conventional group. The experimental group:complete remission 12 cases, significant response 18 cases, improvement 6 cases, the total effective rate was $90.0 \%$; the conventional group:complete remission 5 cases, significant response 9 cases, improvement 14 cases, the total effective rate was $70.0 \%$.It showed that the clinical treatment effect of the experimental group was significantly better than that of the conventional group,and the difference was statistically significant, so it had statistical significance $(\mathrm{P}<0.05)$. See Table 1 below.

Table 1. Analysis of clinical treatment effect of patients in both groups $(n, \%)$

\begin{tabular}{cccccc}
\hline Grouping & $\begin{array}{c}\text { complete } \\
\text { remission }\end{array}$ & $\begin{array}{c}\text { significant } \\
\text { response }\end{array}$ & $\begin{array}{c}\text { improvem } \\
\text { ent }\end{array}$ & invalid & the total effective rate $(\%)$ \\
\hline $\begin{array}{l}\text { conventional } \\
\text { group }(\mathrm{n}=40)\end{array}$ & 5 & 9 & 14 & 12 & 70.0 \\
$\begin{array}{l}\text { experimental } \\
\text { group }(\mathrm{n}=40)\end{array}$ & 12 & 18 & 6 & 4 & $90.0^{*}$ \\
\hline
\end{tabular}

Remark:Compared with the conventional group, ${ }^{*} \mathrm{P}<0.05$

Compared the change of laboratory index of patients in both groups before and after the treatment. After the clinical treatment, the improvement of urine red blood cell count and $24 \mathrm{~h}$ urine protein quantitative of the experimental group were significantly better than that of the conventional group. There was significant difference between two groups, which had statistical significance $(\mathrm{P}<$ 0.05). See Table 2, table 3.

Table 2. Analysis of laboratory index values of patients in both groups before the treatment $(\bar{\chi} \pm S)$

\begin{tabular}{ccccc}
\hline Grouping & $\begin{array}{c}\text { Urine red blood } \\
\text { cell count } \\
(\text { one } / \mathrm{HP})\end{array}$ & $\begin{array}{c}\text { 24h urinary protein } \\
(\mathrm{g} / 24 \mathrm{~h})\end{array}$ & $\begin{array}{c}\mathrm{SCr} \\
(\mu \mathrm{mol} / \mathrm{L})\end{array}$ & $\begin{array}{c}\mathrm{BUN} \\
(\mathrm{mmol} / \mathrm{L})\end{array}$ \\
\hline $\begin{array}{c}\text { conventional } \\
\text { group } \\
\text { experimental } \\
\text { group }\end{array}$ & $17.41 \pm 12.35$ & $1.62 \pm 1.02$ & $133.23 \pm 22.66$ & $6.82 \pm 2.16$ \\
\hline
\end{tabular}

Table 3. Analysis of laboratory index values of patients in both groups before the treatment $(\bar{\chi} \pm S)$

\begin{tabular}{ccccc}
\hline Grouping & $\begin{array}{c}\text { Urine red blood } \\
\text { cell count } \\
(\text { one/HP })\end{array}$ & $\begin{array}{c}\text { 24h urinary protein } \\
(\mathrm{g} / 24 \mathrm{~h})\end{array}$ & $\begin{array}{c}\mathrm{SCr} \\
(\mu \mathrm{mol} / \mathrm{L})\end{array}$ & $\begin{array}{c}\mathrm{BUN} \\
(\mathrm{mmol} / \mathrm{L})\end{array}$ \\
\hline $\begin{array}{c}\text { conventional } \\
\text { group } \\
\text { experimental } \\
\text { group }\end{array}$ & $3.09 \pm 7.16$ & $0.92 \pm 0.87$ & $125.77 \pm 20.43$ & $6.78 \pm 2.23$ \\
\hline
\end{tabular}

Remark:Compared with the conventional group, ${ }^{*} \mathrm{P}<0.05$

\section{Discussion}

IgA nephropathy is one of the common primary glomerulonephritis in clinical practice. Western medicine believe that the pathogenesis of this disease is very complex, which is related to genetic factors, abnormal glycosylation, immune regulation and $\operatorname{IgA} 1$ receptors. While traditional Chinese believed that IgA nephropathy belonged to "drown", "hematuria", "consumptive disease", "pain" [9], mainly due to deficiency of liver-yin and kidney-yin, deficiency of both qi and yin, damp-heat toxin, stagnant blood block,etc.

The main clinical manifestations of this disease were hematuria, proteinuria (with or without), and the incidence of the disease was increasing year by year. Many scholars had carried on the research and discussion to this kind of disease. Clinical research ${ }^{[8]}$ showed that the abnormal immune 
response in $\operatorname{IgA}$ nephropathy played an important role in the pathogenesis of this disease. The fundamental cause of the development of proteinuria, hematuria and others was the result of immune injury, which made a series of changes of the structure of the basement membrane of the patients. The basement membrane could effectively maintain the patient's glomerular structure, and fix the adjacent cells of the patients effective, to constitute the filtration barrier.

In the research, the patients of experimental group carried on clinical treatment of salf-made Shenqi two thistles Er Zhi decoction. The formula composition including Radix Astragali, Radix Pseudostellariae, setose thistle, field thistle, early Lotus grass, glossy privet fruit, Radix stephaniae tetrandrae (TET), Achyranthes root, madder, Gorgon fruit, Rosa laevigata, in them,Radix Pseudostellariae,and Radix Astragali could invigorating qi for strengthening superficies, inducing diuresis to alleviate edema. According to modern medical research, Radix Astragalus could effectively improve microcirculation of patients, red blood cell aggregation and anti platelet, etc., and lower the patients' blood viscosity, increase blood flow, the effective removal of peroxide, and repair glomerular basement membrane. The setose (field)thistle, madder can play a good effect of cooling blood to stop bleeding, promote blood circulation to remove blood stasis, modern pharmacology that, madder can effectively promote the patient thromboplastin generation (thrombin, fibrin). Ligustrum lucidum, early Lotus grass had effect of nourishing the liver and kidney, nourishing yin and tonifying essence; Achyranthes, Radix stephaniae tetrandrae can reach the effect of expelling wind and removing dampness and promoting blood circulation to remove blood stasis, Rosa laevigata, Gorgon can reach the effect of reinforce the kidney, astringent ${ }^{[10]}$. Harmonic using a variety of prescription, can play the effect of Invigorating qi and nourishing Yin, promoting blood circulation and removing blood stasis, expelling wind and dampness, the effect of specimens.

The research results showed that after the treatment of self-made Shenqi two thistles Er Zhi decoction for the patients in the experimental group, the clinical symptoms of patients were significantly improved, the total effective rate was high, reaching $90.0 \%$, while the total effective rate of conventional group using the traditional way of anticoagulation, anti infection and others was $70.0 \%$. There was significant difference between the two groups $(\mathrm{P}<0.05)$; in addition, the urine red blood cell number and $24 \mathrm{~h}$ urinary protein level of the patients in the experimental group were significantly improved after the treatment, and the difference was significant $(\mathrm{P}<0.05)$. It showed that the clinical effect of Shenqi two thistles Er Zhi decoction in the treatment of IgA nephropathy patients was significant, and the clinical symptoms and signs of the patients were significantly relieved, and the clinical significance was significant, so it was worth being promoted and applied vigorously.

\section{References}

[1] G.X. He, S.M. Zheng, Y.P. Ye and others. Self designed Shenqi two thistles Er Zhi decoction in the treatment of 46 cases of clinical study of IgA nephropathy.Chinese Journal of Traditional Medical Science and Technology,2014,21(4):429,431.

[2] W.J. Chen, Y.Y. Deng, X.W. Zhang and others.Clinical study on invigorating spleen and kidney Tongluo recipe in the treatment of asdthenic splenonephro-yang and blood stasis type and progressive IgA nephropathy .Chinese Journal of Integrated Traditional and Western Nephrology,2012,13(10):876-878.

[3] S.H. Wang, Z.Y. Lu, X. Zhang and others. Analysis on etiologt and pathogenesis of IgA nephropathy.Jilin traditional Chinese Medicine,2012,32(7):652-653.

[4] J.R. Lin. Research progress of IgA nephropathy in Chinese Medicine.Medical Journal of Western China,2013,25(2):317-318.

[5] X.R. Wang, Y.X. Wan, Z.H. Zhao and others.Research on the microcosmic syndrome differentiation of TCM syndrome of IgA nephropathy .Chinese Information on Traditional Chinese Medicine, 2015, 22(8): 31- 35. 
[6] H.Z. Ma, H.B. Chen,He Lingzhi and others. Clinical curative effect observation of adding and subtracting Shenqi Dihuang Decoction in treatment of "deficiency of both qi and yin"of $\operatorname{IgA}$ nephropathy. Journal of Zhejiang Chinese Medicine University, 2012,36(5):491-493,496.

[7] M. Chen, Y.X. Wan,Dai Enlai and others. The research of TCM syndrome classification of IgA nephropathy based on the factor analysis and cluster analysis.Journal of Beijing University of Traditional Chinese Medicine,2014,37(2):135-140.

[8] Y. Zhang, L.Z. He.Traditional Chinese medicine treatment of IgA nephropathy.Journal of Changchun University of Traditional Chinese Medicine,2015,31(2):272-275.

[9] Z.H. Zhao, Y.X. Wan,Jiangmin and others.Syndrome classification of IgA nephropathy and correlation analysis of kidney immune deposits.Acta Chinese Medicine and Pharmacology,2012,40(3): 71-73.

[10] Z.Z. Liu, S.X. Lin, X.S. Liu and others.System evaluation of Chinese patent medicine with immunosuppressive effect in the treatment of IgA nephropathy.Chinese General Practice,2012,15(2): 134-138. 